\title{
A bonding process between grains in mechanically disaggregated snow
}

\author{
Edward E. ADAMS, Steven M. JEPSEN, Bryan CLOSE \\ Department of Civil Engineering, Room 205 Cobleigh Hall, Montana State University-Bozeman, \\ MT 59717-3900, USA \\ E-mail: eda@ce.montana.edu
}

\begin{abstract}
Collections of disaggregated snow particles were examined in a temperature-controlled microscope stage. In addition to necks that appeared to sinter in a manner congruent with the twoparticle model, there also appeared unanticipated dendritic growth, which developed on some grains and grew into the pore space. These branches developed preferentially only on part of, and in different directions on, individual grains. Some of these grew enough to join with adjacent grains that were in close proximity but not initially in contact, while the surface of the adjacent grains did not show measurable growth or loss. Growth orientation is hypothesized to be due to crystal habit dependence on temperature. Columnar growth was observed at $-5^{\circ} \mathrm{C}$ and plate-like at $-15^{\circ} \mathrm{C}$. The random growth orientation is in contrast to observed source and sink development aligned with a temperature gradient imposed using a gradient stage. In this case, a source-to-sink directionality across the pore was apparent in which faceted crystals grew at the expense of neighboring source grains. The process of mechanically disaggregating snow produces numerous broken shards and sharp-edged fracture surfaces. We hypothesize that it is the sublimation of these high-surface-energy regions that provides the excess vapor to facilitate the diffusion-limited dendritic growth observed in this 'equitemperature', mechanically processed snow.
\end{abstract}

\section{INTRODUCTION}

Individual snow crystals form in clouds when supercooled, supersaturated water vapor nucleates as ice onto a particulate. In this environment, crystals may develop a wide variety of shapes, but all with a basic hexagonal structure, as demonstrated by Bentley and Humphreys (1931). In a classic study, Nakaya (1954) determined a relationship between the dominant crystallographic growth direction and temperature. From $0^{\circ} \mathrm{C}$ to $\sim-4{ }^{\circ} \mathrm{C}$ plates develop (fastest growth in the plane of the a axes); then down to $\sim-10^{\circ} \mathrm{C}$ columns grow ( $c$ axis fastest); a transition back to plates is observed down to $\sim-22^{\circ} \mathrm{C}$, below which columnar crystals again occur. Secondary features (e.g. branching and dendrites on plates, or needles and hollow sheaths on columns) develop when the supersaturation level exceeds that of saturation over liquid water at the given temperature (Lock, 1990). Branching growth on isolated crystals grown from the vapor begins when the crystal growth is dominated by water-vapor particle diffusion. This type of growth is most notably observed at $-5^{\circ} \mathrm{C}$ where needle (c-axis) growth is dominant and at $-15^{\circ} \mathrm{C}$ where branching along the a axis is most apparent. Complexity increases with the level of supersaturation (Libbrecht, 2005).

When the crystals collect as a snow cover, dynamic morphologic alterations ensue. In a dry snow cover, depending largely on temperature gradient, one of two general processes dominates (de Quervain, 1958; Akitaya, 1974). Larger temperature gradients $\left(>20^{\circ} \mathrm{C} \mathrm{m}^{-1}\right)$ lead to what Colbeck (1982) called the kinetic growth form. The process is particularly prevalent near a relatively warm base, in which case the resulting structure is termed depth hoar (Seligman, 1936). Lower temperature gradients yield a structure composed of rounded, sintered grains, referred to by Colbeck (1982) as the equilibrium growth form. These processes are also often referred to as 'temperature gradient' and 'equitemperature' metamorphism, respectively, although actual equal temperature conditions, as implied by the term equitemperature, are unlikely. Macroscopically, the sintered snow is, in general, structurally stronger.

Sintering is a mechanism that is concerned with the consolidation of powdered materials, resulting in densification, grain growth and microstructural changes. It is commonly exploited in engineering applications in ceramics and powder metallurgy. Although sintering dynamics are quite complex, insight into the process is obtained by considering two spheres in contact. In this two-particle model, the amount of material supplied to the neck (or bond) region is largely based on geometry. Forces that govern sintering in this model result from surface curvature variation, which influences differences in bulk pressure, vacancy concentration and vapor pressure. Kang (2005) lists six material transport mechanisms: (1) lattice diffusion from the grain boundary to the neck, (2) grain boundary diffusion from the grain boundary to the neck, (3) viscous flow from the bulk grain, (4) surface diffusion from the particle surface to the neck, (5) lattice diffusion from the particle surface to the neck and (6) gas phase transport by either evaporation/ condensation or gas diffusion. Material supplied from the grain boundary will result in the grains moving closer together, whereas the other sources yield geometric changes but not contraction.

Sintering in snow has most frequently been attributed to vapor transport (Hobbs and Mason, 1964; Maeno and Ebinuma, 1983). However, this mechanism does not account for shrinkage or densification. Colbeck (1998) presents a grain boundary diffusion model for snow, and there is some physical evidence that grain boundary diffusion may play a role in snow sintering (Adams and others, 2001). Although Adams and others' (2001) analysis 
was for a pure ice system, they indicated that impurities would tend to concentrate at grain boundaries and influence the diffusion. Barnes (2003) suggested that the grain boundary ridges observed by Adams and others (2001) may indeed be impurities and offered that, while not required, the presence of impurities is in accord with grain boundary diffusion and that soluble impurities could increase the diffusion rate. Rosenthal and others (2007) demonstrated evidence that the ridges are impurities and discuss the fact that dopants are used in enhancing engineered sintering processes.

In the present study, we examine microstructural metamorphism of disaggregated snow particles in the absence of an imposed temperature gradient. Microstructure and intergranular bonding between snow grains is of importance to avalanche stability, over-snow mobility, conductivity and other thermomechanical properties. A strong macrostructure is often observed to rapidly develop in snow that has been mechanically worked by mechanisms such as wind, avalanches, snowplows and snowmillers, etc. (Abele, 1990). Causal processes that have been attributed to this include mechanical heat dissipation, which produces slight melt followed by refreeze between grains, and particle sintering mechanisms.

\section{METHODS}

The impetus of this study was to observe sintering in processed snow. Older, rounded grained natural snow that had been stored in an insulated container in a $-10^{\circ} \mathrm{C}$ freezer for several months comprised the initial constituent. Sample preparation was carried out in a cold laboratory near the temperature of the intended metamorphism study. Snow was disaggregated by sifting through a $0.99 \mathrm{~mm}$ sieve into a $16 \mathrm{~mm}$ diameter, $2 \mathrm{~mm}$ tall crucible. The vessel used in most of the studies was constructed by affixing a stainless-steel ring to a thin glass plate. Steel was chosen to assist in achieving a uniform sample temperature. Immediately after snow was sifted into the crucible, it was transferred onto a temperature-controlled silver block, which is the core of the enclosed microscope cold chamber. A glass cover slip was placed over the crucible to facilitate sample temperature uniformity and minimize vapor exchange. In this paper, we refer to these cases as 'equitemperature' experiments.

For the short transfer distance to the microscope, which was outside the cold laboratory, samples were placed in a small insulated container filled with snow and cold packs. It should be noted that the cold-stage chamber must be briefly opened to place the sample, causing a perturbation in the stage environment.

The Linkam BCS196 microscope stage uses liquid nitrogen injected directly into the silver block by a LPN94/2 cooling pump which is controlled by a TMS94 temperature programmer. Temperature feedback is provided by a 1/10 DIN class B Pt100 platinum resistor to give $<0.01 \mathrm{~s}$ response time. A second cryostage was also used in two of the experiments discussed here. The Linkam GS120 is a temperature gradient stage that can be used to program gradients across a sample by using two aligned Peltierbased thermal elements. These elements both lie on the focal plane of the microscope, separated by a $2.5 \mathrm{~mm}$ gap. Each individual element can be independently controlled to $0.1^{\circ} \mathrm{C}$ from -25 to $120^{\circ} \mathrm{C}$ to establish a temperature gradient across the stage, transverse to the microscope observation orientation.

Metamorphism was observed over the course of 2-4 hours, and time-lapse images recorded using a Nikon Eclipse 80i microscope with a Qimaging Retiga-2000R digital camera. The charge-coupled device has 1600 by 1200 square $(7.4 \mu \mathrm{m} \times 7.4 \mu \mathrm{m})$ pixels. The viewing area was selected to examine grain-to-grain and grain-to-pore interaction. This utilized a $100 \times$ microscope magnification to yield a $1.4 \mu \mathrm{m}$ resolution. An effort was made to focus between the upper grains into a region below the top surface to achieve conditions more representative of the interior of a natural snow cover.

In general, the samples were back-lit through a small hole in the silver block or between the Peltier elements. The light intensity was kept to a minimum, to reduce its potential thermal influence, and compensated with exposure time. For the same reason, the exterior of the cryostage was preconditioned by placing cold packs on top, prior to beginning an observation sequence.

As is typical of experimental protocols, the design described evolved through nuanced refinements as efforts were made to eliminate conditions that might unintentionally influence the outcome. Consequently, although all of the experiments did not strictly adhere to all aspects of the protocol presented, the resultant unexpected metamorphism (described below) was reasonably consistent. The question remained, however, as to whether this development was an artifact of the scale of the experiment and irrelevant to larger systems.

To examine a somewhat more in situ type scenario, without boundary influence, a separate procedure was conducted. Instead of being sifted directly into the crucible, snow was sifted through the $0.99 \mathrm{~mm}$ sieve to $70 \mathrm{~mm}$ depth in an $85 \mathrm{~mm}$ diameter cylindrical paper cup that remained undisturbed in the cold laboratory for 4 hours. A sample was then taken from the center of the specimen, placed in the crucible, inserted into the cryostage and immediately observed, presumably prior to additional metamorphism. The stage was set to $-30^{\circ} \mathrm{C}$ in an effort to slow further metamorphism in this 'cup' experiment.

\section{OBSERVATIONS AND HYPOTHESES}

As stated, the notion of this study was to observe sintering processes of mechanically processed snow in a controlled thermal environment. Instead of examining two-particle sintering, processes relevant to an assemblage of particles were considered. Unexpectedly, protrusions developed on some surfaces of individual ice grains that were not in contact with other grains. Furthermore, the growth of these was seemingly random in direction on different grains (Fig. 1). Korolev and others (2004), using a thermal diffusion chamber in a similar temperature range to that employed here, have observed the growth of extended features onto isolated, generally smooth spherical ice drops.

The protrusions that developed in the present study are of particular interest because when they were sufficiently close to a neighboring grain they were observed to connect with the adjacent grain. Bonds were thus established, although the grains were not initially in contact. Why did these relatively slender protrusions grow in a preferential direction on a preferred face on individual crystals, yet 

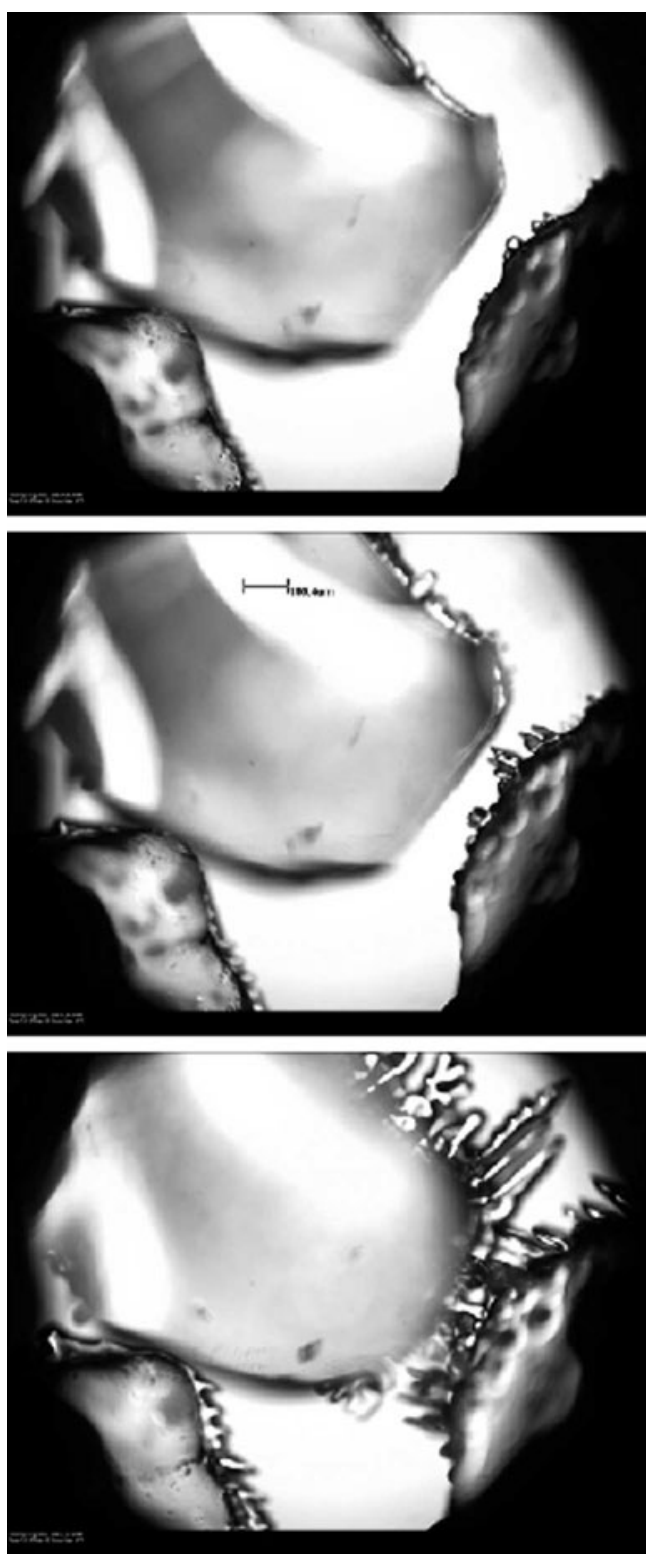

Fig. 1. This top-to-bottom sequence of images demonstrates the development of solid columnar dendrites that were observed at $-5^{\circ} \mathrm{C}$. $\mathrm{C}$-axis ice growth is dominant at this temperature. Protrusions develop only on specific surfaces of the original ice crystals. The growth from the lower right eventually joined with the crystal that occupies the center space in the images. There is neither measurable sublimation nor growth from this surface. Time lapse between the first two images is $20 \mathrm{~min}$ and from the middle to the bottom is 1 hour $56 \mathrm{~min}$. The image sequence was begun when this feature of interest was located: $\approx 20-30 \mathrm{~min}$.

develop a similar morphology but in different directions on other crystals?

Ice crystals grown from the vapor onto an ice substrate in the presence of a temperature gradient will assume the crystallographic orientation of the substrate crystal (Adams and others, 2003), and crystal growth within the snow cover must adhere to the same physics as atmospherically grown crystals. We hypothesize that the selective orientation and habit of the protrusions is governed by the preferred crystallographic morphology dependence on temperature. The needle-like projections observed in Figure 1 developed with the cold stage at $-5^{\circ} \mathrm{C}$. The temperature-dependence hypothesis is supported in observations of a separate
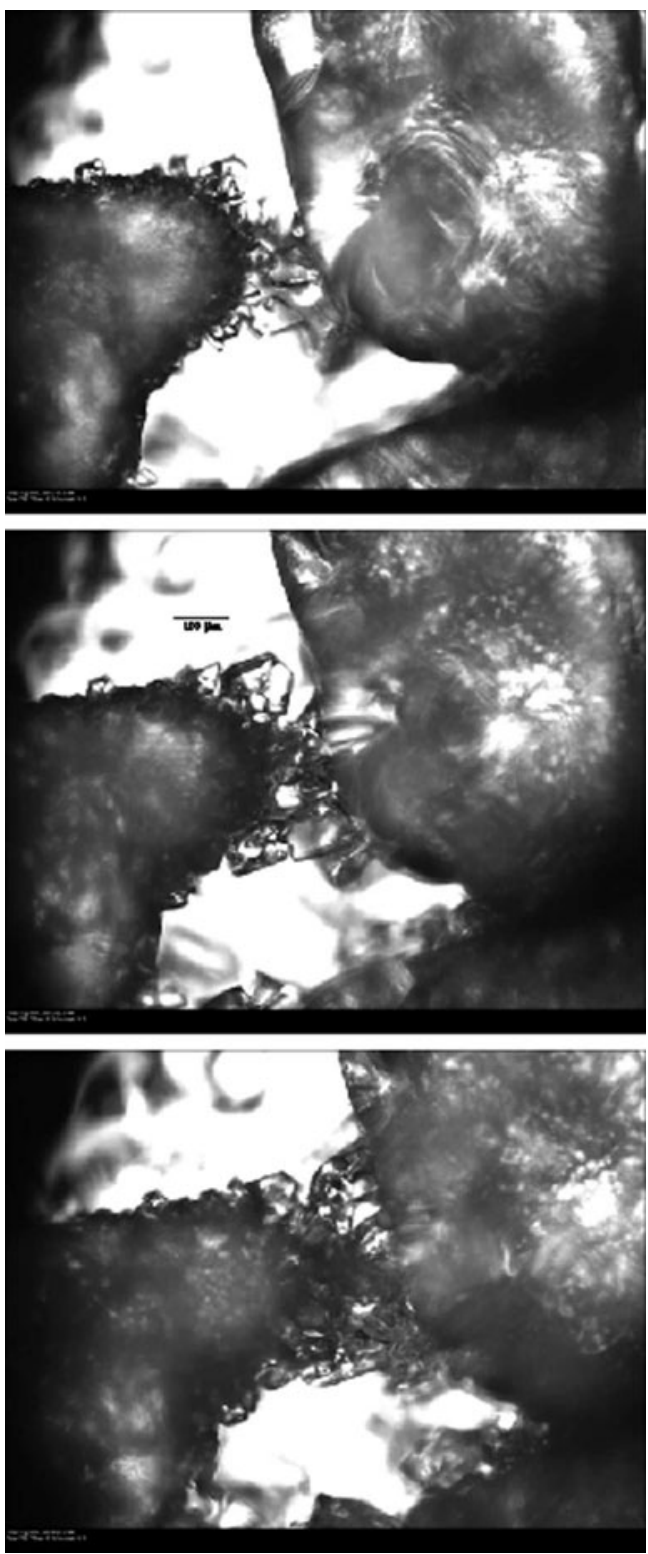

Fig. 2. At $-15^{\circ} \mathrm{C}$, platelets are observed in this top-to-bottom time sequence. A connection is established between two grains. Also note that the branching on the lower left grain appears to develop around the crystal, indicating that the general vantage is likely onto the basal plane. a-axis plates are the dominant ice growth morphology at $-15^{\circ} \mathrm{C}$. The time lapse between the first two images is $35 \mathrm{~min}$ and from the middle to the bottom is 1 hour 5 min. The image sequence was begun when a feature of interest was located.

experiment run at $-15^{\circ} \mathrm{C}$ (Fig. 2) in which the new growth appears to be more plate-like in structure.

There is a potential that these observations might be influenced as the result of a temperature gradient that could develop in the microscope chamber. There is possible variation in the chamber since temperature is controlled by the stage and there is a glass observation port above the sample. It was not possible to determine any preferred growth orientation using the microscope. Therefore, the gradient stage was employed to examine the progressive metamorphism that might be expected in the presence of a prescribed large temperature gradient. The Peltier elements were set to $-5^{\circ} \mathrm{C}$ and $-15^{\circ} \mathrm{C}$, and the ensuing microstructural 

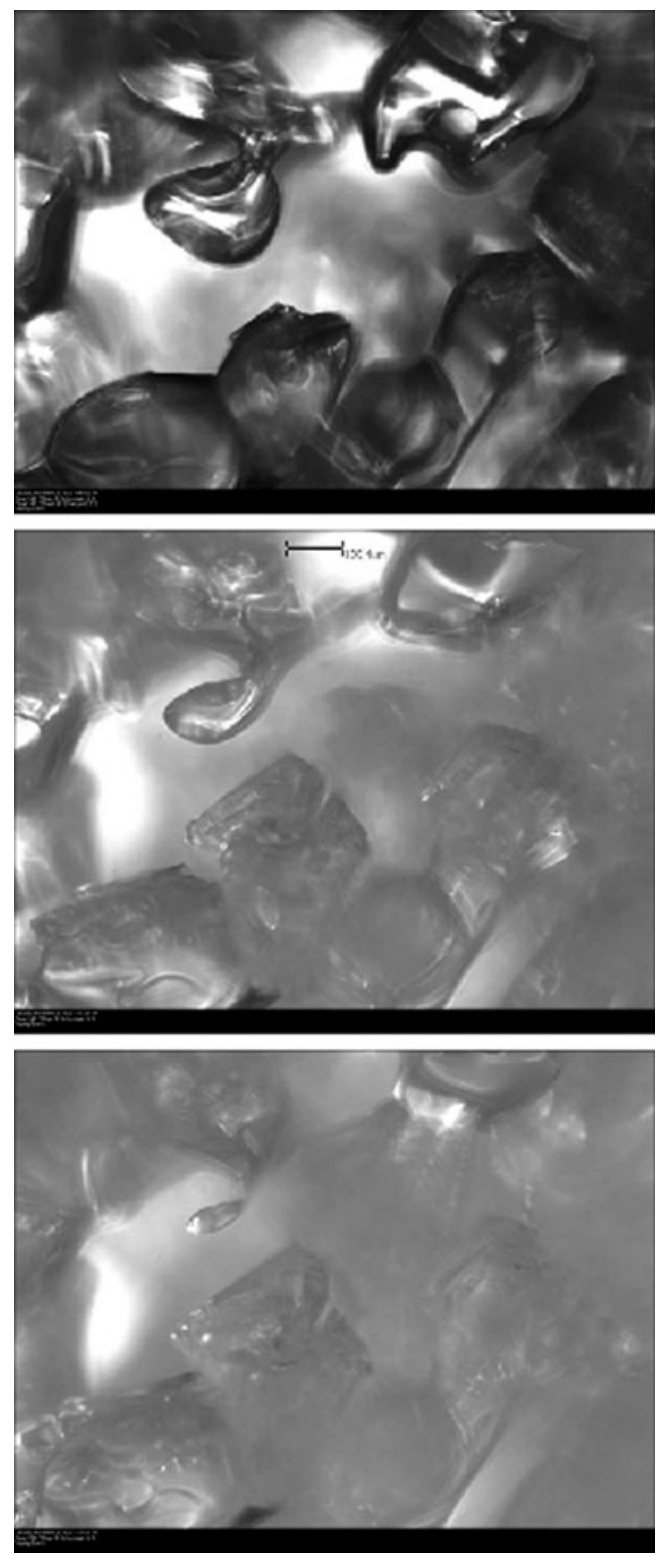

Fig. 3. To contrast growth patterns of the equitemperature with temperature gradient metamorphism, a temperature gradient microscope stage was employed. Two Peltier elements, separated by a $2.5 \mathrm{~mm}$ gap, were set to $-5^{\circ} \mathrm{C}$ and $-15^{\circ} \mathrm{C}$; the warmer temperature toward the top in the image. The snow-filled crucible was placed to span the gap. As expected, directional growth with an exchange of mass across the pore resulted in faceted striated crystals growing at the expense of the warmer grains. The time lapse between the first two images is $25 \mathrm{~min}$ and from the middle to the bottom is $20 \mathrm{~min}$.

changes observed (Fig. 3). A temperature gradient was established in the direction of the crucible diameter. In this environment, faceted striated crystals aligned with the imposed temperature gradient developed at the expense of the adjacent grains. This demonstrates classic temperature gradient metamorphism, described as the 'hand-to-hand' (Yosida and others, 1955) transfer of water vapor across the pore space from the warmer to colder grain.

It is important to note that based on observation in the equitemperature experiments the grain surface to which the new growth joined did not measurably sublimate, nor grow, although on the same grain there was growth at other sections in different directions (Fig. 1).
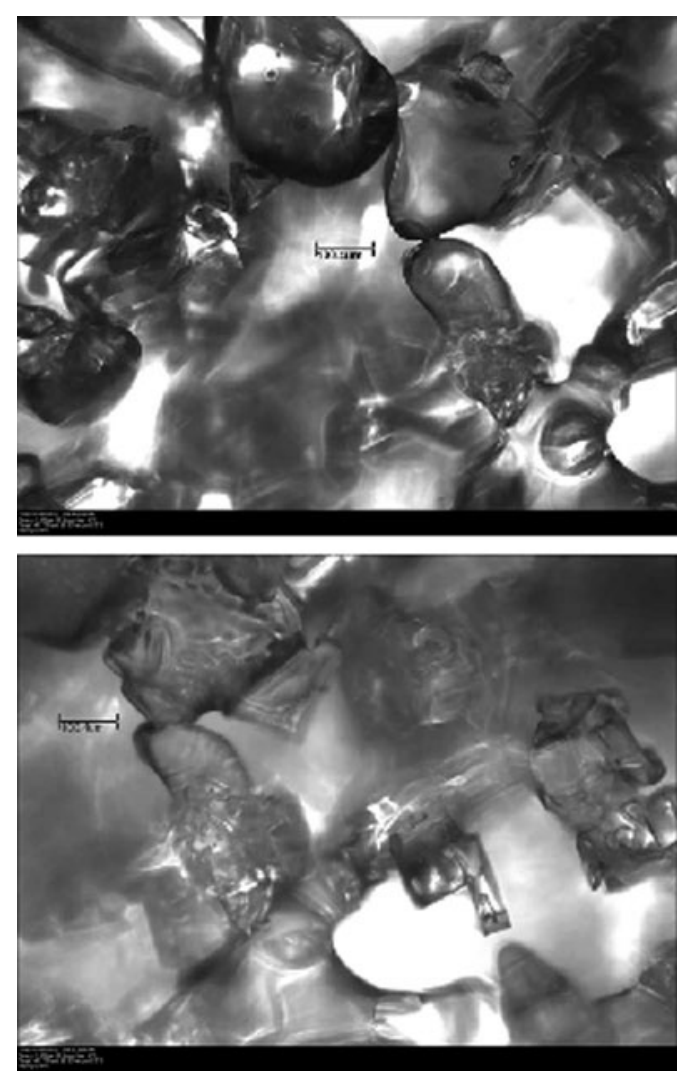

Fig. 4. It was observed in the study that the dendritic protrusions developed simultaneously with sintering grains that were originally in contact. In the top image, sintering has begun for a two-grain contact, just to the right of center. The image on the bottom shows this same bond $39 \mathrm{~min}$ later; the sintering grains are slightly above and left of center. In the lower image, the field of view has been shifted to reveal the columnar structure in the lower right quadrant that developed over this same period. It should be pointed out that the gradient stage with both elements set to $-5^{\circ} \mathrm{C}$ was used. The $0.1^{\circ} \mathrm{C}$ resolution of the elements may have played a role in the morphology of the column which shows signs of faceting and has a slight hollow. This was not observed in the more precise stage.

In the equitemperature studies, the filling-in of necks joining grains which were initially in contact appears to follow the two-particle sintering concept simultaneously with the projection growth (Fig. 4). The column appears in the lower right section of the bottom image. This particular equitemperature experiment was performed using the gradient stage with both elements set to $-5^{\circ} \mathrm{C}$. This observed hollow columnar prism may have been influenced by the $0.1^{\circ} \mathrm{C}$ variations possible in the Peltier elements. This type of hollow faceted development was not noted on samples in the more precise stage.

Examinations of samples taken from the cup experiments indicate that the protrusion growth will also develop in regions further beneath the surface than that which was observed to form in the microscope stage (Fig. 5). However, these protrusions are smaller than those that were observed growing under the cryostage environment for a similar duration. Furthermore, since the protrusions may develop quickly, this demonstration does not preclude the potential that these formed during the first few minutes in the cryostage.

There were instances where the needles grew for a time, but when they did not connect with another particle they 

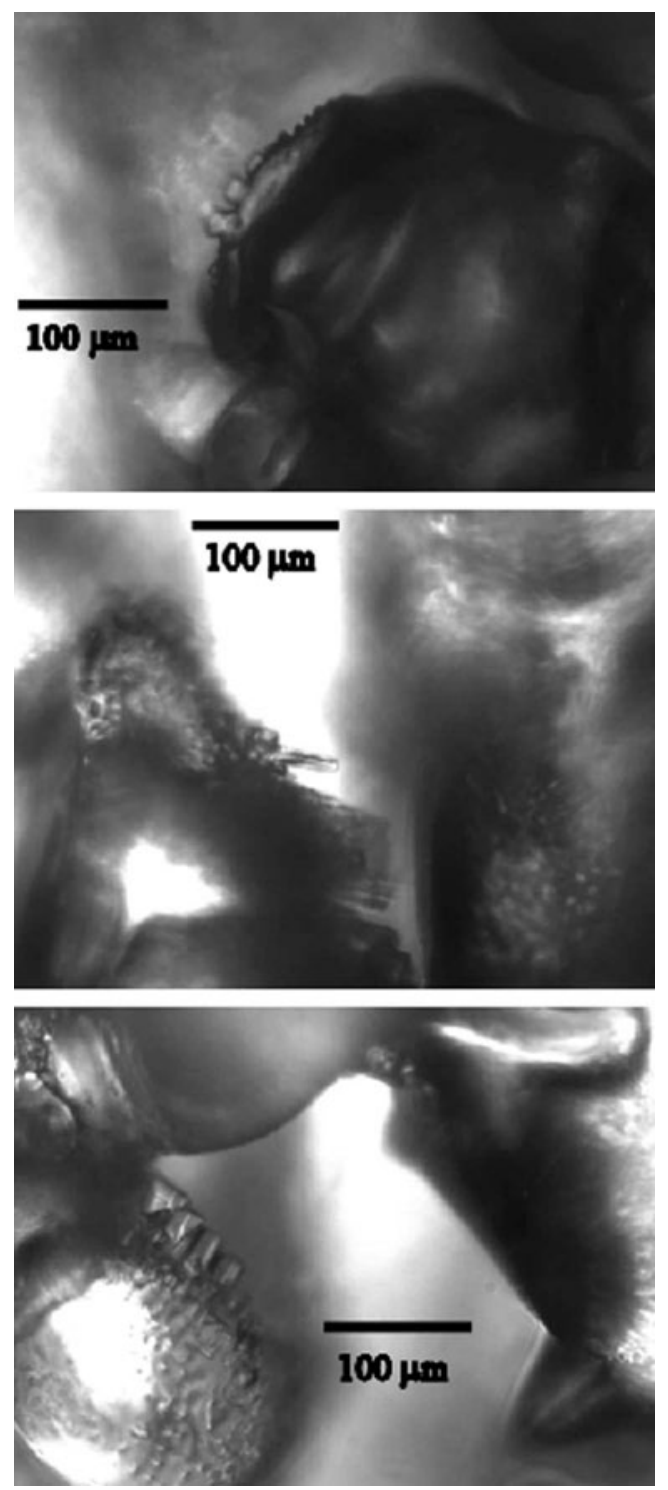

Fig. 5. Snow sifted into an $85 \mathrm{~mm}$ diameter cylinder, $70 \mathrm{~mm}$ deep, in a $-5^{\circ} \mathrm{C}$ cold laboratory remained undisturbed for 4 hours. A specimen was then extracted from the center, placed into the cryostage and immediately observed. Stage temperature was set at $-30^{\circ} \mathrm{C}$ in an effort to slow additional metamorphism. The top image was observed within $3 \mathrm{~min}$ of being placed on the stage; the others were observed 2 and 3 min later.

were observed to retreat. The shallow depth of field of the microscope stage coupled with the metamorphism process over time caused difficulty in maintaining image focus and illumination consistency, making longer-term microscope observations problematic.

\section{DISCUSSION}

Development of the crystal growth from the vapor within a snow cover is examined in this approximation, assuming that the essential physics that determine the growth rate and habit are the same as for atmospherically grown crystals. Although this assumption is utilized here, it should be noted that there are substantive differences between the growth from the vapor of isolated crystals and that which develops in an aggregate such as snow. For example, the concept of the far field and uniform mean vapor distribution would not be the same. In the following analysis, we consider aspects of the summary presented by Libbrecht (2005). Crystal growth is considered in terms of the simple hexagonal prism and the development of the dendritic form.

The velocity of the ice growth normal to the surface, based purely on attachment kinetics-limited growth, is given as (Hertz-Knudsen formula),

$$
v_{\mathrm{n}}=\alpha v_{\text {kin }} \sigma_{\text {surf }}
$$

where $\alpha \leq 1$ is the condensation coefficient (1 if an atom hitting the surface immediately sticks) and $\sigma_{\text {surf }}$ is the supersaturation just above the ice surface.

$$
v_{\text {kin }}=\frac{\rho_{\text {sat }}}{\rho_{\text {solid }}} \sqrt{\frac{k T}{2 \pi m}}
$$

is described by Libbrecht (2005) as the rate at which the surface is simultaneously evaporating and regrowing'. $\rho_{\text {sat }}$ and $\rho_{\text {solid }}$ are the densities for water-vapor equilibrium over a flat ice surface and solid ice respectively, $k$ is Boltzmann's constant, $T$ is temperature and $m$ is the mass of a water molecule. Faceted crystal growth is taken to be attachment kinetics-limited. However, water-vapor particle and heat diffusion also play a role in crystal morphology. When the ice growth velocity is $\ll \alpha v_{\text {kin, }}$ it is considered to be a diffusion-limited process. At $-5^{\circ} \mathrm{C}, v_{\text {kin }}=496 \mu \mathrm{m} \mathrm{s}^{-1}$, which for the approximately constant protrusion growth velocity observed $\left(v_{\text {tip }} \approx 0.024 \mu \mathrm{m} \mathrm{s}^{-1}\right)$ implies that diffusion-limited growth is likely.

A precise development addressing dendritic growth within the snowpack is not yet available; however, some useful insight is presented by utilizing a number of assumptions in the development that is implemented below. Early work in the area of dendritic growth was established by Ivantsov (1947), and a significant body of work has followed.

In air, water-vapor particle diffusion is taken to restrict growth more than does heat diffusion. Libbrecht (2005) considers a particle diffusion model where the velocity and radius of a cylindrically symmetric parabolic dendrite tip, $v_{\text {tip }}$ and $R_{\text {tip }}$, are taken to remain constant. Dendritic tip velocity is approximated as

$$
v_{\text {tip }}=\frac{2 D \alpha s}{R_{\text {kin }} B^{2}} \frac{\rho_{\text {sat }}}{\rho_{\text {solid }}} \sigma_{\infty}
$$

$D=2 \times 10^{-5} \mathrm{~m}^{2} \mathrm{~s}^{-1}$ is the temperature-dependent diffusion constant for water vapor in air, $\alpha$ is the condensation coefficient, $s$ is a dimensionless solvability parameter, $\sigma_{\infty}$ is the supersaturation at a distance ' $f a r^{\prime}$ ' from the growing surface, and $B$ is taken by Libbrecht to be equal to 10 for experimental studies. $R_{\text {kin }}$ is presented as

$$
R_{\text {kin }}=\frac{R_{\text {tip }} \alpha s}{B} .
$$

For molecularly rough surfaces, $\alpha \approx 1$, although accurate values are difficult to determine. $s$, which is taken as a constant, dependent on the intrinsic attachment kinetics, has not yet been calculated. However, in air at $1 \mathrm{~atm}$, $R_{\text {kin }}=30 \mathrm{~nm}$. Therefore if $R_{\text {tip }}$ is known, $\alpha s$ can be estimated from Equation (4). Then through the measured value for the tip velocity, $v_{\text {tip }}$, an approximation of the supersaturation $\left(\sigma_{\infty}\right)$ may be ascertained using Equation (3).

The dendritic growth shown in Figure 1 was examined to obtain a coarse approximation of the tip velocity and radius. These were acquired using a scale (integral to the microscope software) to visually determine and manually 
mark positions on the images with a cursor. Unfortunately, there is considerable subjectivity associated with the method. The ice-boundary edges in the image are not precise, and determination of what constitutes the actual tip radius is inexact. In addition, any slope of the protrusion into or out of the plane will influence the apparent velocity, because the image is based on a projected length. Keeping in mind these difficulties and the limitations of the model, the level of supersaturation in the pores was calculated. The assumption that the tip velocity and radius remain constant appears to be generally consistent with these measurements. Taking as an average $v_{\text {tip }}=0.024 \mu \mathrm{m} \mathrm{s}^{-1}$ and $R_{\text {tip }}=9 \mu \mathrm{m}$ produces a value of $\sigma_{\infty}=0.015$, which yields an excess vapor density (supersaturation density) of $\approx 0.05 \mathrm{~g} \mathrm{~m}^{-3}$. This supersaturation and temperature places us in a region consistent with solid prism growth and below the vapor saturation over supercooled water at $-5^{\circ} \mathrm{C}$ (Libbrecht, 2005).

The significance of the observations noted in this study is the potential relation to the strengthening of processed snow. However, extrapolation of the observations of this study to the large-scale field environment must be scrutinized. Among the potential unintended influences innate to the experimental design are: the constraint to optically focus on subjects slightly below the surface, with the potential of significant surface boundary effects; the perturbation of the cryostage environment when the sample is inserted; heating from the microscope illumination source; and slight temperature gradients that may result from the viewing window above the sample and the cold platform. Measures taken to address these issues, such as minimizing the light intensity, using steel for the vertical cylindrical walls of the crucible, and placing cold packs on the external stage environment, did not appear to alter the observations. Morphologic changes that developed in the presence of an imposed temperature gradient were markedly different from those that developed under the equitemperature conditions. The presence of growth protrusions in the cup experiment, designed to mitigate the influence of the surface boundary, adds credence to the prospect of the protrusion development in a natural environment.

Development of the observed metamorphism that occurs in the experimental conditions and which is potentially relevant to the full-scale environment suggests that, depending upon conditions, a number of processes may play a role in snow sintering. We might speculate that while radius-ofcurvature driven evaporation/condensation processes dominate under some conditions, the dendritic growth discussed here may play a role in some specific situations, and grain boundary diffusion may perhaps play a more obvious role in the longer term.

\section{CONCLUSION}

A conglomeration of disaggregated snow subjected to constant temperature conditions can develop protrusions on individual grains that grow into the interstitial pore space. Growth occurs on specific regions of the ice surface, determined by the crystallographic orientation of the nucleation grain. The preferred growth plane and the crystallographic habit are a consequence of the recognized relationship of temperature with the morphology of ice crystal growth from vapor. It is likely that the observed dendritic development is particle-diffusion limited, with respect to water vapor in air. We hypothesize that the excess vapor density, which drives this process, is due to the availability of the high-surface-energy contours that result from the sieving process employed in disaggregation of the old sintered snow. Fine ice particles and sharp corners that result from fracturing will sublimate, thus providing a mass supply for crystal growth. Connections between two grains can develop when the temperature, the crystallographic orientation of the nucleate grain and the proximity of a neighboring grain are conducive. Protrusions that have connected with another grain may continue to sinter, filling in and strengthening the neck region. But those that do not may disappear when the supply of mass from the fine particles and corners is exhausted and the protrusion tips themselves become regions of relatively high vapor pressure. The dendritic growth assumes the orientation of the crystal from which it is derived; consequently, we conjecture, as sintering progresses, the two originally separate units may assume the morphology of two crystals joined at a single bond interface.

It is well known that snow that has been mechanically worked, such as avalanche debris, wind deposits and snowplows or snowmillers, rapidly strengthens macroscopically (Abele, 1990). Results of the present study suggest that one mechanism in the process may be the growth of dendritic protrusions that grow between and join ice grains. These produce bonds in addition to those that form between grains that are initially in contact and which develop through mechanisms described by the two-particle sintering model. Although this mechanism has intriguing implications, it must be noted that conditions in this study were small-scale and may have been influenced by the boundary and initial conditions of the experimental protocol. Additional work is warranted to validate the relevance of the observed results to field-scale studies.

\section{ACKNOWLEDGEMENTS}

We thank the M.J. Murdock Charitable Trust and the US National Science Foundation (NSF) Award ANT-0521360 for instrumentation to support this work, as well as NSF grant EAR-0635977.

\section{REFERENCES}

Abele, G. 1990. Snow roads and runways. CRREL Monogr. 90-3. Adams, E.E. and D.A. Miller. 2003. Ice crystals grown from vapor onto an orientated substrate: application to snow depth-hoar development and gas inclusions in lake ice. J. Glaciol., 49(164), 8-12.

Adams, E.E., D.A. Miller and R.L. Brown. 2001. Grain boundary ridge on sintered bonds between ice crystals. J. Appl. Phys., 90(11), 5782-5785

Akitaya, E. 1974. Studies on depth hoar. Contrib. Inst. Low Temp. Sci. A26.

Barnes, P.R.F. 2003. Comment on 'Grain boundary ridge on sintered bonds between ice crystals' [J. Appl. Phys., vol. 90, 5782-5785 (2001)]. J. Appl. Phys., 93(1), 783-785.

Bentley, W.A. and W.J. Humphreys. 1931. Snow crystals. New York, etc., McGraw-Hill.

Colbeck, S.C. 1982. Growth of faceted crystals in a snow cover. CRREL Rep. 82-29.

Colbeck, S.C. 1998. Sintering in a dry snow cover. J. Appl. Phys., 84(8), 4585-4589. 
De Quervain, M.R. 1958. On metamorphism and hardening of snow under constant pressure and temperature gradient. IASH Publ. 46 (General Assembly of Toronto 1957 - Snow and Ice), Vol. 4, 225-239.

Hobbs, P.V. and B.J. Mason. 1964. The sintering and adhesion of ice. Philos. Mag., 9(98), 181-197.

Ivantsov, G.P. 1947. Temperature field around a spherical, cylindrical, and needle-shaped crystal, growing in a pre-cooled melt. Dokl. Akad. Nauk SSSR, 58, 567-569.

Kang, S.-J.L. 2005. Sintering: densification, grain growth and microstructure. Oxford, etc., Elsevier Butterworth-Heinemann.

Korolev, A.V., M.P. Bailey, J. Hallett and G.A. Isaac. 2004. Laboratory and in situ observation of deposition growth of frozen drops. J. Appl. Meteorol., 43(4), 612-622.

Libbrecht, K.G. 2005. The physics of snow crystals. Rep. Progr. Phys., 68(4), 855-895.
Lock, G.S.H. 1990. The growth and decay of ice. Cambridge, etc., Cambridge University Press.

Maeno, N. and T. Ebinuma. 1983. Pressure sintering of ice and its implication to the densification of snow at polar glaciers and ice sheets. J. Phys. Chem., 87(21), 4103-4110.

Nakaya, U. 1954. Snow crystals: natural and artificial. Cambridge, MA, Harvard University Press.

Rosenthal, W., J. Saleta and J. Dozier. 2007. Scanning electron microscopy of impurity structures in snow. Cold Reg. Sci. Technol., 47(1-2), 80-89.

Seligman, G. 1936. Snow structure and ski fields. London, Macmillan \& Co.

Yosida, Z. and colleagues. 1955. Physical studies on deposited snow. I. Thermal properties. Contrib. Inst. Low Temp. Sci. A7, $19-74$ 